\title{
Simulation Analysis of Effect of Vacancies on Ferroic Domain Growth of $\mathrm{BaTiO}_{3}$
}

\author{
Takahiro Tsuzuki \\ Graduate School of Engineering \\ Nagoya Institute of Technology \\ Nagoya, Japan \\ t.tsuzuki.794@stn.nitech.ac.jp
}

\author{
Shuji Ogata \\ Graduate School of Engineering \\ Nagoya Institute of Technology \\ Nagoya, Japan \\ ogata@nitech.ac.jp
}

\author{
Ryo Kobayashi \\ Graduate School of Engineering \\ Nagoya Institute of Technology \\ Nagoya, Japan \\ kobayashi.ryo@nitech.ac.jp
}

\author{
Masayuki Uranagase \\ Graduate School of Engineering \\ Nagoya Institute of Technology \\ Nagoya, Japan \\ uranagase.masayuki@nitech.ac.jp
}

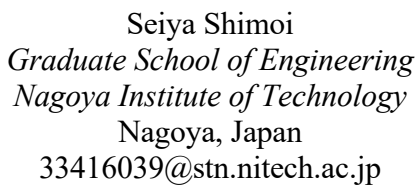

\author{
Saki Tsujimoto \\ Graduate School of Engineering \\ Nagoya Institute of Technology \\ Nagoya, Japan \\ 33412077@stn.nitech.ac.jp
}

Received: June 8, 2021. Received: November 30, 2021. Accepted: December 27, 2021. Published: December 31, 2021.

\begin{abstract}
BaTiO3 is one of the well-known ferroelectric and piezoelectric materials, which has been widely used in various devices. However, the microscopic mechanism of the ferroelectric domain growth is not understood well. We investigated the effects of point defects, mono- and di-vacancies of $\mathrm{Ba}, \mathrm{Ti}$, and $\mathrm{O}$, on the domain growth of BaTiO3 using molecular dynamics simulation with the core-shell inter-atomic potential. We found the following: s (1) One kind of monovacancy, VO1, located on the TiO plane perpendicular to the applied electric field direction, acts to hinder the polarization inversion induced by the applied electric field. The monopole electric field produced by VO1 either hinders or assists the local polarization inversion in accordance with the local intensity of the total electric field. (2) The 1st-neighbor divacancies VBa-VO and VTi-VO as compared to the 2nd-neighbor divacancies asymmetrically affect the domain growth with respect to the applied electric field, making the hysteresis behavior of applied electric field vs. polarization relation. The domain grows even at a small electric field when the directions of the applied electric field and the divacancy dipole are mutually the same. (3) The domain growth speed towards the applied electric field direction is about 2 orders of magnitude higher than that towards the perpendicular direction.
\end{abstract}

Keywords-Barium titanate, point defect, molecular dynamics simulation

\section{INTRODUCTION}

$\mathrm{BaTiO}_{3}(\mathrm{BT})$ is a well-known piezoelectric material, widely used in various devices as, e.g., capacitor for electric circuits, stress sensor, and actuator [1]. The ferroelectric state of BT is, in general, composed of polarized domains whose growths due to applied electric field and stress relate to piezoelectric performance. However, little is known about the atomistic dynamics of ferroelectric domain growth. Essential quantities expected to be evaluated theoretically include the barrier freeenergy for domain boundary shift and the domain wall mobility with or without defects [2], [3].

Depending on the production procedure (e.g., solidification from melt of $\mathrm{TiO}_{2}$ and $\mathrm{BaO}$ ), doping (e.g., La), and external oxygen gas pressure, various point defects are expected to form in BT [4], [5]. The oxygen vacancies $\left(\mathrm{V}_{\mathrm{O}}\right)$ should emerge at a relatively low oxygen pressure. The $\mathrm{V}_{\mathrm{O}}$ was predicted to increase the barrier energy for the domain wall shift by DFT calculations at zero temperature [3]. As for the barium vacancy $\left(\mathrm{V}_{\mathrm{Ba}}\right)$ or titanium vacancy $\left(\mathrm{V}_{\mathrm{Ti}}\right)$ that exists in oxygen-rich $\mathrm{BT}$, the experiment and DFT calculations predicted that such negatively charged monovacancies may combine with positively charged $\mathrm{V}_{\mathrm{O}}$ to form the divacancies $\mathrm{V}_{\mathrm{Ba}}-\mathrm{V}_{\mathrm{O}}$ and $\mathrm{V}_{\mathrm{TI}}-$ $\mathrm{V}_{\mathrm{O}}$ [6]-[9]. The domain growth dynamics in the presence of an applied electric field were studied by the classical molecular dynamics (MD) simulation with or without the points defects. Wang et al. analyzed the effect of $\mathrm{V}_{\mathrm{O}}$ on the hysteresis loop of the applied electric field-polarization relation of BT [10]. Similarly, Ying et al. considered the effect of divacancy $\mathrm{V}_{\mathrm{Ba}}$ $\mathrm{V}_{\mathrm{O}}$ on the hysteresis loop of BT [11]. However, no MD simulation about the effect of divacancy $\mathrm{V}_{\mathrm{Ti}}-\mathrm{V}_{\mathrm{O}}$ on the domain growth has been reported as far as we know.

Motivated by that we aim to comprehensively analyze the effects of mono- and di-vacancies on the domain growth of BT by using the MD simulation with the core-shell inter-atomic potential. We consider not only the monovacancies $\mathrm{V}_{\mathrm{Ba}}, \mathrm{V}_{\mathrm{Ti}}$, and $\mathrm{V}_{\mathrm{O}}$ but also the divacancies of 1st- and 2nd-neighbor $\mathrm{V}_{\mathrm{Ba}^{-}}$ $\mathrm{V}_{\mathrm{O}}$ and $\mathrm{V}_{\mathrm{Ti}}-\mathrm{V}_{\mathrm{O}}$. We also compare the domain growth speed between parallel and perpendicular directions to the applied electric field.

\section{SimUlATION METHODS AND SETTINGS}

We use the core-shell inter-atomic potential [12] in the MD 
simulation. The core-shell potential mimics the valence electron polarization of an atom by decomposing it to a core for the nucleus or ion and a shell for the valence electrons (see Fig. 1(a)). The cores and shells of atoms in the simulation system interact with each other through the anharmonic spring potential

$$
\phi^{\mathrm{S}}\left(\left|\boldsymbol{r}_{i j}\right|\right)=\frac{1}{2} k_{2}\left|\boldsymbol{r}_{i j}\right|^{2}+\frac{1}{24} k_{4}\left|\boldsymbol{r}_{i j}\right|^{4}
$$

Buckingham potential

$$
\phi^{\mathrm{B}}\left(\left|\boldsymbol{r}_{i j}\right|\right)=A \exp \left(-\frac{\left|\boldsymbol{r}_{i j}\right|}{\rho}\right)-\frac{c}{\left|\boldsymbol{r}_{i j}\right|^{6}},
$$

and Coulomb potential

$$
\phi^{\mathrm{C}}\left(\left|\boldsymbol{r}_{i j}\right|\right)=\frac{q_{i} q_{j}}{\left|\boldsymbol{r}_{i j}\right|} .
$$

Here $k_{2}, k_{4}, A, \rho$, and $c$ are adjustable parameters, and $\left|\boldsymbol{r}_{i j}\right|=$ $\left|\boldsymbol{r}_{i}-\boldsymbol{r}_{j}\right|$ with $\boldsymbol{r}_{i(j)}$ the position of core/shell- $i(j)$. The charge of core/shell $-i$ is $q_{i}$. In the present study, values of the core-shell parameters are taken from Vielma et al. [13], which well reproduce the temperature-dependent polarization behavior, lattice phase, and lattice constants of BT.

In our original simulation code, we explicitly calculate the long-ranged Coulomb interaction of charges under the periodic boundary conditions (PBCs) using the Ewald method. The shell positions are determined at each timestep for core positions so that the total potential energy takes the minimum value.

We prepare the simulation system under PBCs as $16 \times 16 \times$ 16 unit cells of BT; numbers of atoms are 4,096 for both Ba and $\mathrm{Ti}$ and 12,288 for $\mathrm{O}$. The tetragonal unit cell size in perovskite structure has side lengths $(4.0,4.05,4.0) \AA$ in $(x, y, z)$ direction at temperature $300 \mathrm{~K}$ considering the polarization in $y$-direction; that is, $1 \%$ elongation in $y$-direction (see Fig. 1(b)). The MD simulations are performed in the NVT ensemble. We use the Langevin thermostat method to maintain the system temperature at around $300 \mathrm{~K}$. The timestep is $3 \mathrm{fs}$, and the time evolution is conducted with the velocity-Verlet method.

First, the system is equilibrated for $10 \mathrm{ps}$ at $300 \mathrm{~K}$ to a randomly polarized state. Second, we apply the electric field of $-60 \mathrm{MV} / \mathrm{m}$ in $y$-direction for $10 \mathrm{ps}$ to form a fully polarized single domain in $-y$-direction. Third, we calculate the hysteresis loop by gradually changing the electric field from $-60 \mathrm{MV} / \mathrm{m}$ to $60 \mathrm{MV} / \mathrm{m}$ and then to $-60 \mathrm{MV} / \mathrm{m}$ with the time rate of $\pm 0.1 \mathrm{MV} / \mathrm{ps}$ or $\pm 0.05 \mathrm{MV} / \mathrm{ps}$ (particularly for sensitive stage). Considering thermal fluctuations in the system, we repeat the simulations for maximumly 20 loops.

To create a monovacancy in the system, we remove an atom from the perfect crystal; it corresponds to $0.024 \%$ of unit cells having a vacancy. We set a uniform background charge to keep the charge neutrality of the system. A monovacancy of

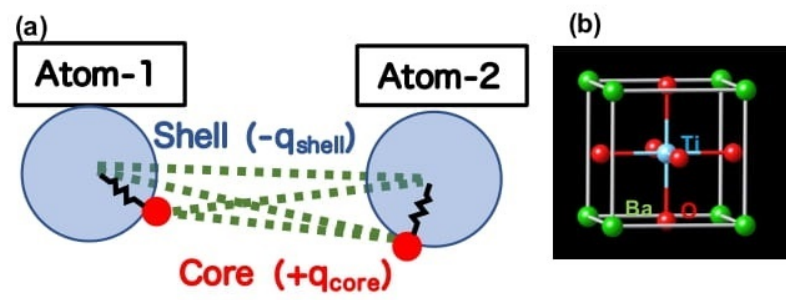

Fig. 1. (a) Schematic view of the core-shell inter-atomic potential. (b) Tetragonal unit cell of $\mathrm{BaTiO}_{3}$ in perovskite structure.

$\mathrm{Ba}$ (Ti) at any location is equivalent to the lattice symmetry; that is, $\mathrm{V}_{\mathrm{Ba}(\mathrm{Ti})}$ is one kind. On the other hand, an $\mathrm{O}$ monovacancy has two kinds: $\mathrm{V}_{\mathrm{O} 1}$ and $\mathrm{V}_{\mathrm{O} 2}$ are respectively located on $\mathrm{TiO}$ and $\mathrm{BaO}$ planes perpendicular to the $y$-direction (see, Fig. 2(a)).

The divacancy, created by removing two mutually neighboring atoms from the perfect crystal, is particularly interesting because it has the intrinsic dipole due to negatively charged $\mathrm{V}_{\mathrm{Ba}(\mathrm{Ti})}$ and positively charged $\mathrm{V}_{\mathrm{O}}$, which may effectively magnify the applied electric field depending on the dipole direction. We, therefore, consider those divacancies that have positive $y$-components of the intrinsic dipoles. As for the 1st-neighbor divacancy, both $\mathrm{V}_{\mathrm{Ba}}-\mathrm{V}_{\mathrm{O}}$ and $\mathrm{V}_{\mathrm{Ti}}-\mathrm{V}_{\mathrm{O}}$ have one kind as depicted in Fig. 2(b). Fig. 2(c) shows the 2nd-neighbor divacancies $\mathrm{V}_{\mathrm{Ba}}-\mathrm{V}_{\mathrm{O}}$ and $\mathrm{V}_{\mathrm{Ti}}-\mathrm{V}_{\mathrm{O}}$ with two kinds: $\mathrm{V}_{\mathrm{Ba}}-\mathrm{V}_{\mathrm{O}}$ and $\mathrm{V}_{\mathrm{Ti}}-\mathrm{V}_{\mathrm{O}}$ with $\mathrm{V}_{\mathrm{O} 1}$ on a $\mathrm{TiO} x z$-plane, $\mathrm{V}_{\mathrm{Ba}}-\mathrm{V}_{\mathrm{O} 2}$ and $\mathrm{V}_{\mathrm{Ti}}-\mathrm{V}_{\mathrm{O} 2}$ with $\mathrm{V}_{\mathrm{O} 2}$ on a $\mathrm{BaO} x z$-plane.

\section{RESUltS AND DISCUSSIONS}

\section{A. Development of modified conjugate gradient $(C G)$ minimization method}

For the present simulation with the core-shell inter-atomic potential, we modified the CG minimization method, which is used at each timestep to determine the shell positions for instantaneous core positions so that the total potential energy takes the minimum value. The original CG minimization method is composed of iterative calculations with respect to conjugate direction and minimum-value position along the
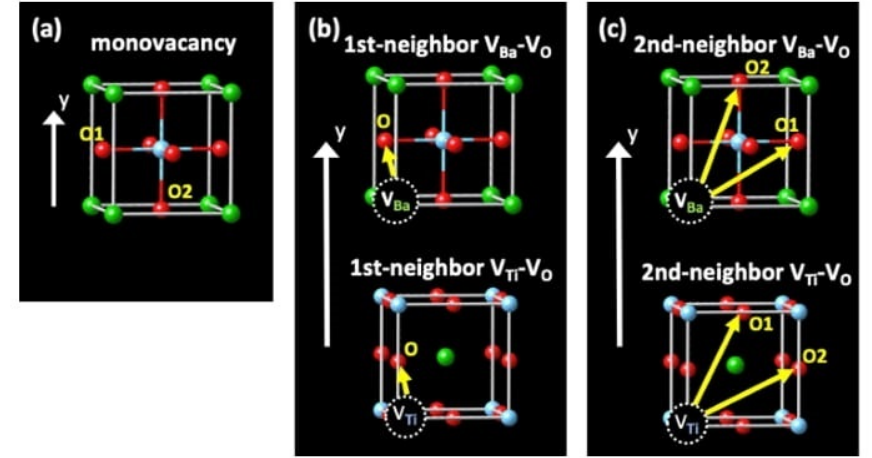

Fig. 2. (a) Definitions of two kinds of oxygen monovacancies. The $\mathrm{V}_{\mathrm{O} 1}$ is located on $\mathrm{TiO}$ plane perpendicular to the spontaneous polarization $(y$ direction), while $\mathrm{V}_{\mathrm{O} 2}$ is located on $\mathrm{BaO}$ plane. (b) Definitions of 1st-neighbor $\mathrm{V}_{\mathrm{Ba}}-\mathrm{V}_{\mathrm{O}}$ and $\mathrm{V}_{\mathrm{T}}-\mathrm{V}_{\mathrm{O}}$ divacancies, which have one kind. (c) Definitions of 2ndneighbor $\mathrm{V}_{\mathrm{Ba}}-\mathrm{V}_{\mathrm{O}}$ and $\mathrm{V}_{\mathrm{Ti}}-\mathrm{V}_{\mathrm{O}}$, which have two kinds. 
direction.

In [12], the minimization was done after approximating the target function as effectively quadratic one by using the function values at three positions $\left\{\lambda_{0}, \lambda_{1} \lambda_{2}\right\}$ along a search direction. The minimum-value position $(\lambda)$ of the quadratic function is analytically obtained. Therefore, three times of potential energy calculations per CG iteration are needed. Considering that the potential energy calculation is the most time-consuming part, we propose a modified CG method where only one time of potential energy calculation per CG iteration is needed, as follows.

In the modified CG method, we first use only the quadratic spring-term of Eq. (1), which is a leading term of the total potential energy in most situations, in each CG iteration and analytically obtain the minimum-value position $\left(\lambda_{1}\right)$ of the term. We then regard the minimum-value position $\lambda_{\text {quad,improved }}$ of the total potential energy as $\lambda_{\text {quad,improved }}=$ $\alpha \lambda_{1}$ with a heuristically pre-determined factor $\alpha$. Only a single time of potential energy calculation is needed per CG iteration in this method. To determine $\alpha$, we compare required computation timings by varying $\alpha$. Fig. 3 shows the mean computation timing per MD step vs. $\alpha$. The timing is nearly proportional to the required number of $\mathrm{CG}$ iterations to reach the minimum potential energy. As seen in Fig. 3, the timing is minimum at $\alpha=0.65$. When $\alpha>0.8$, the shell sometimes cannot reach the minimum potential energy positions in this method.

To understand the reason why $\alpha=0.65$ is appropriate, we calculate the exact minimization position $\lambda$ in unit of $\lambda_{1}$, that is, $\alpha$, using the bisection minimization method. We parenthetically note that the accurate minimum finding method such as the bisection method requires several tens times of function calculations per CG iteration; that is, too timeconsuming to be used in the MD simulation. Histogram of exact $\alpha$ thereby obtained for various simulation settings and CG iteration stages is shown in Fig. 4. As seen in Fig. 4, the histogram assumes the largest frequency at around $\alpha=0.65$ in accordance with the $\alpha$ for the minimum mean computation timing in Fig. 4.

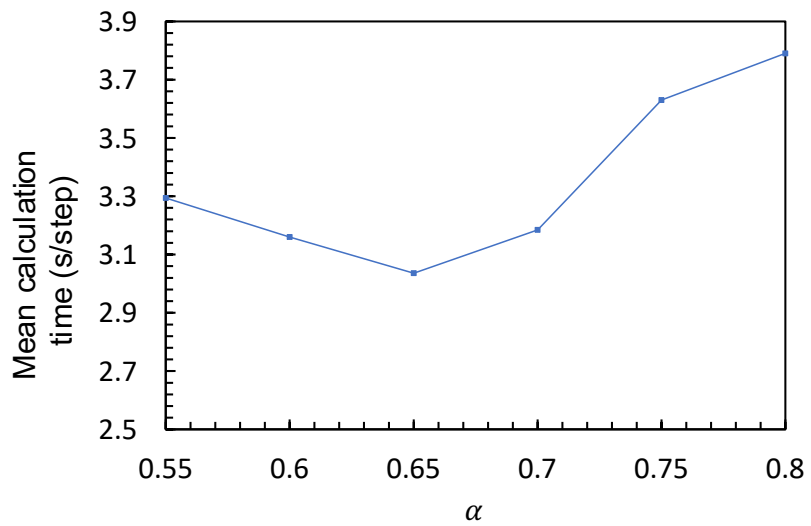

Fig. 3. Mean calculation timing per MD step as a function of factor $\alpha$ in the present MD simulation.

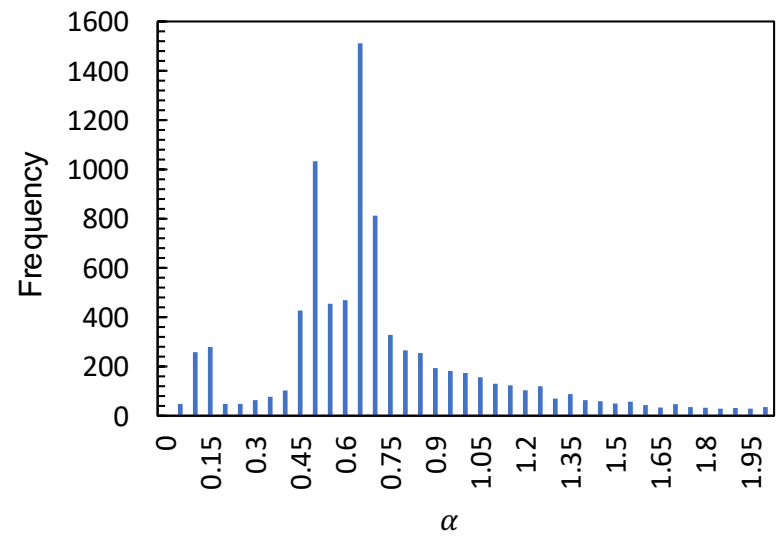

Fig. 4. Histogram of exact $\alpha$ obtained for various simulation settings and CG iteration stages.

The modification of the CG iteration explained above works well in the present MD code; we set $\alpha=0.6$. Depending on the potential energy terms, the modification may not be applicable or the factor $\alpha$ need be changed dynamically.

\section{B. Hysteresis loop}

Fig. 5 shows the results of hysteresis loops obtained in the MD simulation runs at $300 \mathrm{~K}$ for the perfect crystal, $\mathrm{V}_{\mathrm{Ba}}-\mathrm{V}_{\mathrm{O}}$, and $\mathrm{V}_{\mathrm{Ti}}-\mathrm{V}_{\mathrm{O}}$ cases. Substantial fluctuations of the loop are observed particularly for the perfect crystal. Red curves in Fig. 5 corresponds to the averaged loop over the loops in the repeating runs (grey curves).

For the monovacancy cases, we find no substantial deviation of the loop from that of the perfect crystal. In the cases of the 1st-neighbor divacancy, the hysteresis loop shows asymmetry with respect to the positive and negative applied electric field, while no substantial asymmetry, in the 2nd-neighbor divacancy cases. When we apply the electric field anti-parallel to the 1stneighbor divacancy dipole, the polarization starts inverting at nearly the same electric field strength as in the case of the perfect crystal. On the other hand, when we apply the electric field parallel to the 1st-neighbor divacancy dipole, the polarization starts inverting at a substantially smaller electric field, indicating acceleration of polarization inversion due to the divacancy. Such acceleration is more significant for $\mathrm{V}_{\mathrm{Ba}}-\mathrm{V}_{\mathrm{O}}$ than for $\mathrm{V}_{\mathrm{Ti}}-\mathrm{V}_{\mathrm{O}}$ as seen in Fig. 5(b) and (c).

We calculate the polarization of each unit cell, which we call the local polarization. Initially, all the local polarizations of the system, which fluctuates in directions and magnitudes, have $-y$-components because of the negative electric field in the $y$-direction. After the applied electric field is changed to $+y$-direction, such local polarizations have increased probabilities to have $+y$-components. We show in Fig. 6 the probability of each local polarization having $+y$-component in the simulation runs of the systems with 1st-neighbor $\mathrm{VBa}_{\mathrm{Ba}} \mathrm{V}_{\mathrm{O}}$ and $\mathrm{V}_{\mathrm{Ti}}-\mathrm{V}_{\mathrm{O}}$ under an applied electric field of $10 \mathrm{MV} / \mathrm{m}$. Each closed circle in Fig. 6 corresponds to a local polarization with its color representing the probability value. 

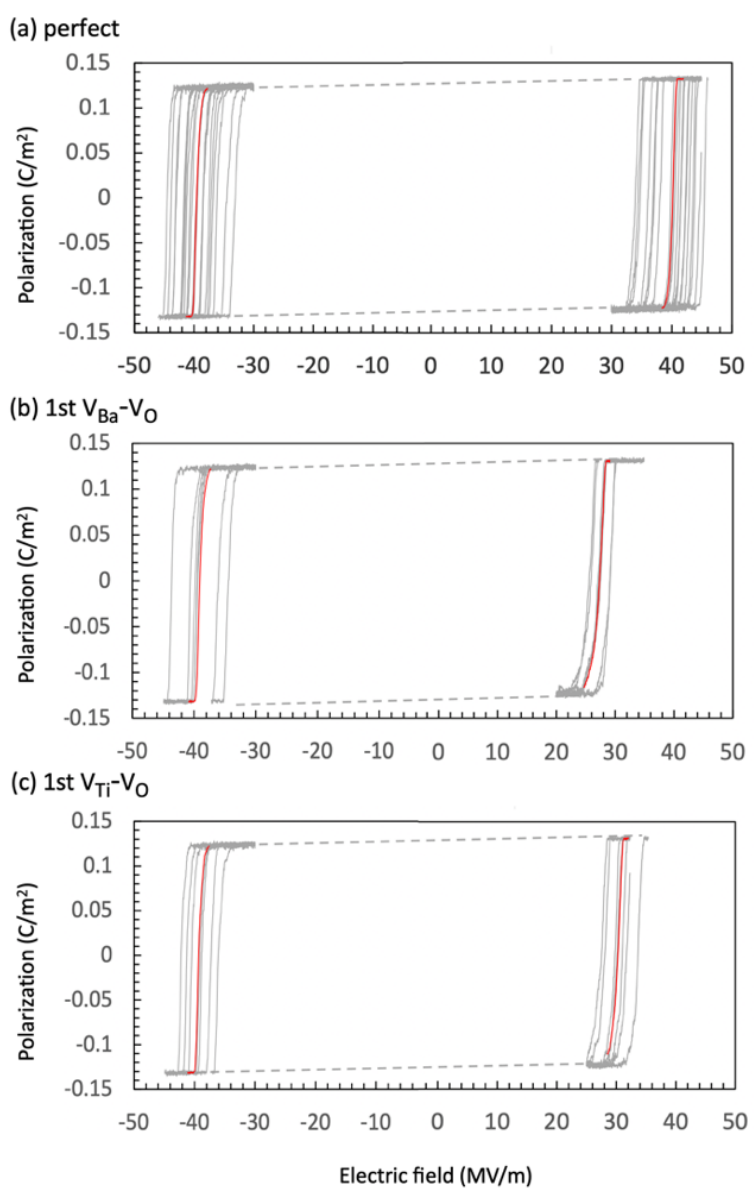

Fig. 5. The hysteresis loops of the applied electric field vs. system polarization calculated by the MD simulation runs. (a) for perfect crystal, (b) for 1stneighbor $\mathrm{V}_{\mathrm{Ba}}-\mathrm{V}_{\mathrm{O}}$ divacancy, and (c) for 1 st-neighbor $\mathrm{V}_{\mathrm{Ti}}-\mathrm{V}_{\mathrm{O}}$ divacancy.

Substantially increased probabilities of having $+y$ component of local polarization are observed in Fig. 6 for both $\mathrm{V}_{\mathrm{Ba}}-\mathrm{V}_{\mathrm{O}}$ and $\mathrm{V}_{\mathrm{Ti}}-\mathrm{V}_{\mathrm{O}}$ divacancies. It is because the divacancy dipole has $+y$-components and hence locally enhances the electric field (see Fig. 2). Relatively small increase in the probability on the $+y$ side of the $y$-column on $\mathrm{V}_{\mathrm{Ti}}-\mathrm{V}_{\mathrm{O}}$ is observed in Fig. 6(b) for the $\mathrm{V}_{\mathrm{Ti}}-\mathrm{V}_{\mathrm{O}}$ case, in comparison to the $\mathrm{V}_{\mathrm{Ba}}-\mathrm{V}_{\mathrm{O}}$ case. It is in accordance with the limited acceleration in the polarization inversion due to $\mathrm{V}_{T i}-\mathrm{V}_{O}$ in Fig. 5(c). Detailed analyses on the origin of such differences between $\mathrm{V}_{\mathrm{Ba}}-\mathrm{V}_{\mathrm{O}}$ and $\mathrm{V}_{\mathrm{Ti}}-\mathrm{V}_{\mathrm{O}}$ are in progress.

\section{Domain growth speed}

Time evolution of the inverted domain is depicted in Fig. 7 for a run of 1st-neighbor VBa-VO located at the center. In the run, an embryo of the inversion emerges around the divacancy in the form of y-column. It then continuously grows in size. In another run of 1st-neighbor $\mathrm{VBa}-\mathrm{VO}$, the inverted domain shrinks after some degree of growth. Such varying behaviors

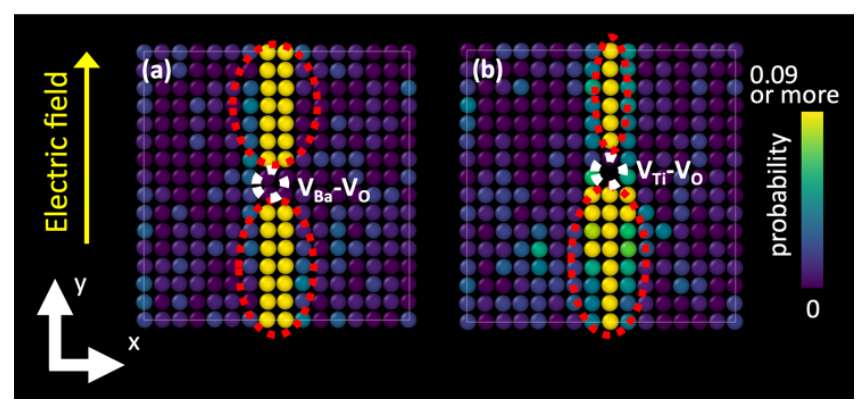

Fig. 6. The probability for the local polarization having positive y-component when electric field of $10 \mathrm{MV} / \mathrm{m}$ is applied in $+y$-direction to fully $-\mathrm{y}$-polarized system. (a) for system with $\mathrm{V}_{\text {Ba-vo }}$ and (b) for system with $\mathrm{V}_{\mathrm{Ti}} \mathrm{i} \mathrm{V}_{\mathrm{O}}$. The white dashed circle at the center depicts the location of divacancy. Divacancy dipole has a positive y- component.

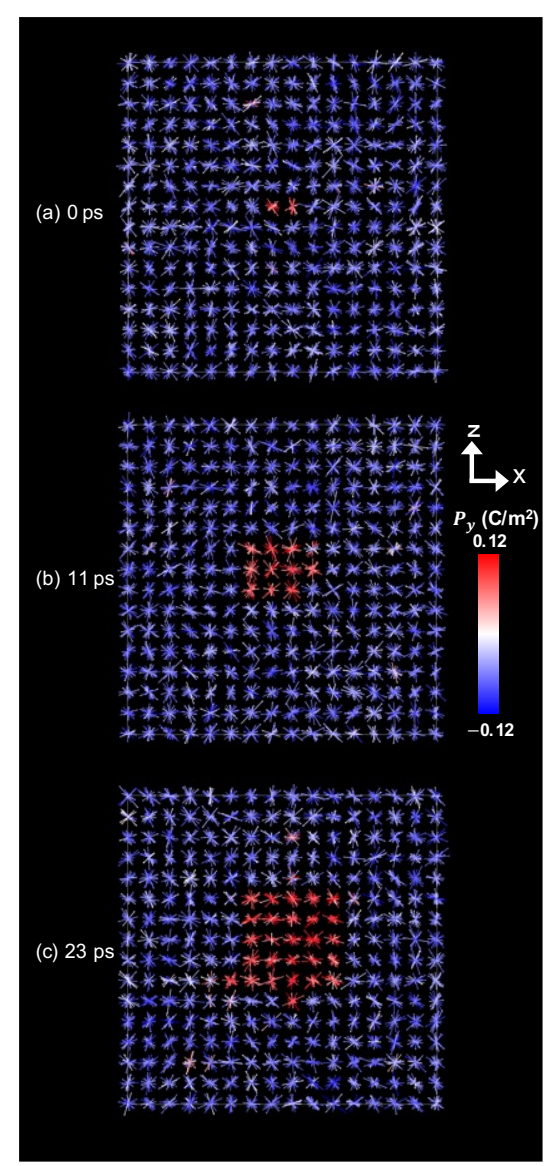

Fig. 7. Snapshots of polarization inversion process in the MD simulation for the case of 1 st-neighbor $\mathrm{V}_{\mathrm{Ba}}-\mathrm{V}_{\mathrm{O}}$. The $\mathrm{V}_{\mathrm{Ba}}-\mathrm{V}_{\mathrm{O}}$ is locate at the ceter.

among the runs are reasonable because the homogeneous nucleation theory predicts an energy barrier for domain growth from the competition of the positive interface energy and the negative energy of inverted phase. Such an energy barrier is stochastically surpassed by fluctuating thermal energy. We evaluate the growth speed in $x z$-direction about $10^{-3} \AA /$ fs.

Fig. 8 shows the time evolution of local polarizations in a single $y$-column of unit cells in the simulation run for a perfect crystal with the electric field $-40 \mathrm{MV} / \mathrm{m}$ applied in the $y$ direction. All local polarizations have $+y$-components (red 


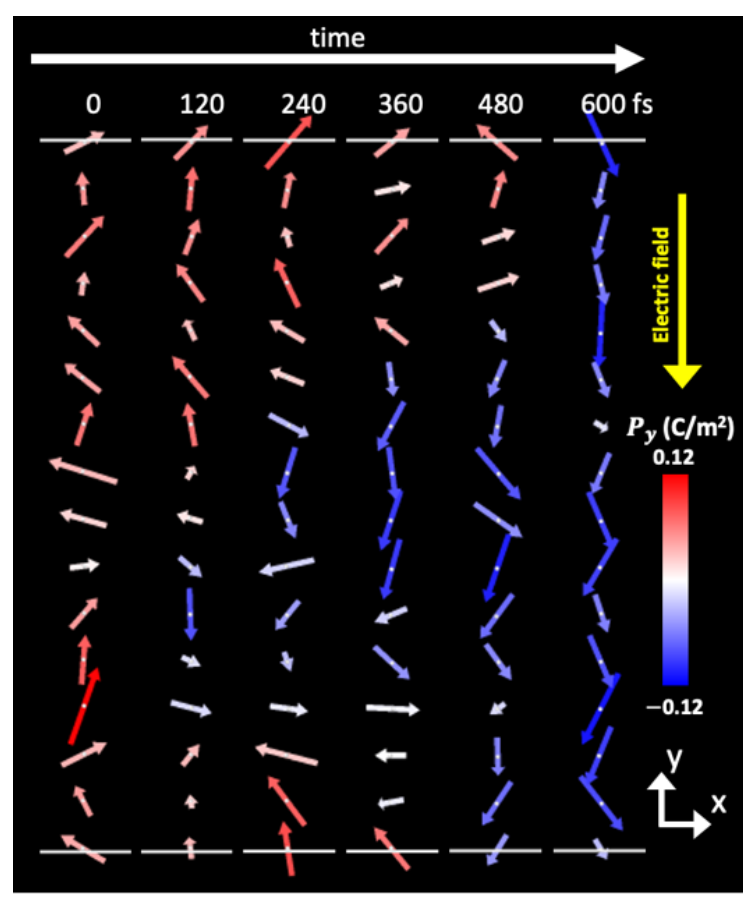

Fig. 8. Time evolution of the local polarizations in a $y$-column when an electric field of $-40 \mathrm{MV} / \mathrm{m}$ in $y$-direction is applied to the fully $+y$-polarized system. Each arrow indicates the local polarization of a unit cell. Its color represents the sign of $y$-component.

means positive in Fig. 8) at 0 fs. Then, local polarizations gradually change their directions to have $-y$-components (i.e., blue in Fig. 8) from the central area of the $y$-column. All the local polarizations in the $y$-column are fully inverted at $600 \mathrm{fs}$. $\mathrm{We}$, therefore, evaluate the domain growth speed in the $y$ direction as $0.1 \AA / \mathrm{fs}$. We state that the domain growth speed in the $y$-direction (i.e., the applied electric field direction) is about two orders of magnitude larger than that in the $x z$-direction.

\section{CONCLUSION}

We investigated the effect of points defects, mono and divacancies of $\mathrm{Ba}, \mathrm{Ti}$, and $\mathrm{O}$, on the hysteresis loop of $\mathrm{BaTiO}_{3}$ by performing the classical molecular dynamics simulation. We found: (1) The 1st-neighbor divacancies $\mathrm{V}_{\mathrm{Ba}}-\mathrm{V}_{\mathrm{O}}$ and $\mathrm{V}_{\mathrm{Ti}}-\mathrm{V}_{\mathrm{O}}$ as compared to the 2nd-neighbor divacancies affect more the domain growth under the applied electric field in an asymmetrical manner with respect to its direction, making the hysteresis behavior of applied electric field vs. polarization relation. (2) The domain starts growing at a smaller electric field for $\mathrm{V}_{\mathrm{Ba}}-\mathrm{V}_{\mathrm{O}}$ than for $\mathrm{V}_{\mathrm{Ti}}-\mathrm{V}_{\mathrm{O}}$ when the directions of the applied electric field and the divacancy dipole are mutually the same. (3) The domain growth speed towards the applied electric field direction is about two orders of magnitude higher than that towards the perpendicular direction.

\section{ACKNOWLEDGMENT}

This work was supported by the JSPS Japanese-German Graduate Externship. The computations were performed using Fujitsu FX1000 at the information technology center of Nagoya University, the supercomputer system at Research Institute for Information Technology of Kyushu University, and highperformance intercloud at the Information Initiative Center of Hokkaido University.

\section{References}

[1] M. Acosta, N. Novak, V. Rojas, S. Patel, R. Vaish, J. Koruza, G. A. Rossetti, and J. Rödel, "BaTiO3-based piezoelectrics: Fundamentals, current status, and perspectives," Applied Physics Reviews, vol. 4, no. 4, p. 041305, Dec. 2017.

[2] V. Boddu, F. Endres, and P. Steinmann, "Molecular dynamics study of ferroelectric domain nucleation and domain switching dynamics," Sci. Rep., vol. 7, no. 1, p. 806, Dec. 2017.

[3] X. Y. Li, Q. Yang, J. X. Cao, L. Z. Sun, Q. X. Peng, Y. C. Zhou, and R. X. Zhang, "Domain Wall Motion in Perovskite Ferroelectrics Studied by the Nudged Elastic Band Method," $J$ Phys. Chem. C, vol. 122, no. 5, pp. 3091-3100, 2018.

[4] Y. Feng, J. Wu, Q. Chi, W. Li, Y. Yu, and W. Fei, "Defects and Aliovalent Doping Engineering in Electroceramics," Chem. Rev., vol. 120, no. 3, pp. 1710-1787, Feb. 2020.

[5] C. L. Freeman, J. A. Dawson, H.-R. Chen, L. Ben, J. H. Harding, F. D. Morrison, D. C. Sinclair, and A. R. West, "Energetics of donor-doping, metal vacancies, and oxygen-loss in A-site rareearth-doped BaTiO3," Adv. Funct. Mater., vol. 23, no. 31, pp. 3925-3928, Aug. 2013.

[6] A. M. Massoud, R. Krause-Rehberg, H. T. Langhammer, J. Gebauer, and M. Mohsen, "Defect studies in BaTiO3 ceramics using positron annihilation spectroscopy," in Materials Science Forum, 2001, vol. 363, pp. 144-146.

[7] B. Liu, Y. Ma, Y. Zhou, and J. Ding, "Single Vacancies and Vacancy Pairs in Tetragonal BaTiO3: A First Principles Study," Ferroelectrics, vol. 401, no. 1, pp. 36-44, Oct. 2010.

[8] J. N. Baker, P. C. Bowes, J. S. Harris, and D. L. Irving, "Mechanisms governing metal vacancy formation in $\mathrm{BaTiO} 3$ and SrTiO3," J. Appl. Phys., vol. 124, no. 11, 2018.

[9] P. Erhart and K. Albe, "Thermodynamics of mono-and divacancies in barium titanate," J. Appl. Phys., vol. 102, no. 8, 2007.

[10] J. Wang, Y. G. Shen, F. Song, F. J. Ke, Y. L. Bai, and C. S. Lu, "Effects of oxygen vacancies on polarization stability of barium titanate," Sci. China. Ser. G: Phys. Mech. Astron., vol. 59, no. 3, pp. $1-4,2016$.

[11] Y. Ma, B. Liu, Y. Zhou, and J. Ding, "Alignment of Ba-O divacancies as a mechanism for polarization imprint in $\mathrm{BaTiO} 3$ as revealed by first principles and classical molecular dynamics simulations," Appl. Phys. Lett., vol. 96, no. 12, pp. 3-6, 2010.

[12] P. J. D. Lindan and M. J. Gillan, "Shell-model molecular dynamics simulation of superionic conduction in CaF2," J. Phys. Condens. Matter, vol. 5, no. 8, pp. 1019-1030, Feb. 1993.

[13] J. M. Vielma and G. Schneider, "Shell model of BaTiO3 derived from ab-initio total energy calculations," J. Appl. Phys., vol. 114, no. $17,2013$.

\section{Creative Commons Attribution License 4.0 (Attribution 4.0 International, CC BY 4.0)}

This article is published under the terms of the Creative Commons Attribution License 4.0 https://creativecommons.org/licenses/by/4.0/deed.en_US 